\title{
3D Lane Detection System Based on Stereovision
}

\author{
Sergiu Nedevschi, Rolf. Schmidt, Thorsten Graf, Radu Danescu, \\ Dan Frentiu, Tiberiu Marita, Florin Oniga, Ciprian Pocol
}

\begin{abstract}
This paper presents a 3D lane detection method based on stereovision. The stereovision algorithm allows the elimination of the common assumptions: flat road, constant pitch angle or absence of roll angle. Moreover, the availability of $3 \mathrm{D}$ information allows the separation between the road and the obstacle features. The lane is modeled as a 3D surface, defined by the vertical and horizontal clothoid curves, the lane width and the roll angle. The lane detection is integrated into a tracking process. The current lane parameters are predicted using the past parameters and the vehicle dynamics, and this prediction provides search regions for the current detection. The detection starts with estimation of the vertical profile, using the stereo-provided 3D information, and afterwards the horizontal profile is detected using a model-matching technique in the image space, using the knowledge of the already detected vertical profile. The roll angle is detected last, by estimating the difference of the average heights of the left and right lane borders. The detection results are used to update the lane state through Kalman filtering.
\end{abstract}

\section{INTRODUCTION}

$\mathrm{L}^{\mathrm{A}}$ ANE detection is a key problem in any driving assistance system, and one of the privileges of computer vision. Laser scanners and radars may detect the obstacles faster and more accurately, but the lane delimiters must be somehow seen in a graphical way. The problem of detecting the lane may seem very simple, especially in highway scenarios, when the road markings are clear and the lane has a well-defined geometry. The reasons for which this problem may seem simple is that very often the accuracy of the algorithm is judged by the quality of the detection in the image, and not by the quality of the lane geometry parameters and of the ego car positioning

Manuscript received April 1, 2004. This work was supported by Volkswagen A.G., Germany

S. Nedevschi, R. Danescu, D. Frentiu, T. Marita, F. Oniga, C. Pocol are with Technical University of Cluj-Napoca, Computer Science Department, 400020 Cluj-Napoca, ROMANIA (phone: +40-264-401457. fax: +40-264-594491; e-mail: Sergiu.Nedevschi@cs.utcluj.ro).

R. Schmitd, T. Graf are with Volkswagen AG, Elecironic Research, 38436 Wolfsburg, GERMANY (phone: +49-5361-9-75316, +49-5361-9 29420, fax: +495361 9-72837; email: rolf4.schmidt@volkswagen.de, thorsten.graf@volkswagen.de) parameters. These parameters are crucial for a driving assistance system. An error of one meter in detection of an obstacle's position may not be very severe, especially if the object is not very close, but an error of a tenth of a degree in estimation of the ego vehicle's position inside the lane may lead to an incorrect trajectory prediction, when traveling at highway cruise speed. The situation is made even more serious by the fact that the lane is usually used as a reference system for object tracking.

Lane detection has been for quite a long time the monopoly of the monocular image processing techniques. Monochrome images tend to be preferred over color, due to better resolution and reduced data load. Lane detection methods become in this case a problem of fitting a certain $2 \mathrm{D}$ model to the image data. The 2D model can be a projection of a 3D road model (a clothoid curve is the most commonly used 3D model) in the image plane [1], [2], [3], [4], [5], [6], [7], [8], usually the projection of a flat road of constant curvature, or it can be any mathematical model which can be matched under some robustness constraints, such as splines or snakes [9], [10]. Some methods try to transform the $2 \mathrm{D}$ image into a surrogate $3 \mathrm{D}$ space by using inverse perspective mappings, under certain assumptions [11], [12], [13], [14]. Sometimes the model itself is not explicitly stated, but it is implied by some assumptions that the algorithm takes (for instance, parallel lines).

The data to which the model is fitted can be the grayscale image, which can undergo several enhancements [11], the image gradient [1], [2], [10], [3], features extracted using frequency domain processing [15], edges extracted using specialized edge detectors [8], or higher level features, such as the whole road marking [16].

The models are fitted to the image using different techniques, many of which involve some kind of search and a likelihood function to be maximized [1], [10], [14], an energy function to be minimized [9], the use of the Hough transform [8] or the iterative update of a probability model using a Kalman filter [3], [7], [17]. A somewhat different method, using a multi-agent architecture approach is presented in [12].

After the lane is detected in the image the 3D information can be extracted in several possible ways. If the $2 \mathrm{D}$ model 
is a projection of the 3D one, the parameters of the 3D lane can be easily extracted, provided that a calibration was initially performed. Under certain assumptions, road 3D information can be extracted from the image [5]. 3D information can also be extracted using the Kalman filter and regarding the 3D lane parameters as the state vector and the $2 \mathrm{D}$ detection as the measurement [3], [7], [17]. 3D information can be extracted also by inverse perspective mapping (IPM) [11]. Reference [13] presents a technique that also detects the pitch angle from the relative orientation of the lane markings in the IPM image. Information from other types of sensors, such as radar, can be fused into the lane detection process [2] for better 3D results.

All the monocular lane detection methods suffer from their connection to a specific assumption. The detection itself is based on these assumptions (flat road, constant curvature, etc), and when it is the time to extract 3D information the assumptions take an even heavier toll.

This paper presents a lane detection method based on stereovision. The stereovision algorithm allows the elimination of the assumptions of flat road, constant pitch angle or absence of roll angle (which is actually the most common of all assumptions). Moreover, the availability of 3D information allows the separation between the road and the obstacle features. The lane is modeled as a $3 \mathrm{D}$ surface, defined by the vertical and horizontal clothoid curves, the lane width and the roll angle, and it is detected by modelmatching techniques.

\section{LANE MODEL PARAMETERS}

Lane detection is regarded as the continuous estimation of the following parameters [18]:

- $W$ - the width of the lane

- $\quad c_{h, 0}$ - horizontal curvature of the lane

- $\quad c_{h, I}$ - variation of the horizontal curvature of the lane

- $c_{v, 0}$ - vertical curvature of the lane

- $X_{c w}$ - the lateral displacement of the car reference system from the lane reference system (lane center)

- $\quad \alpha, \psi, \psi$ the pitch, roll and yaw angles of the car (the rotation angles between the car reference system and the world reference system).

These parameters describe the lane position and geometry through the following equations:

$$
\begin{aligned}
& X_{c}=-X_{c w^{\prime}}-\psi Z+c_{0, h} \frac{Z^{2}}{2}+c_{1, h} \frac{Z^{3}}{6} \\
& X_{L}=X_{c}-\frac{W}{2} \\
& X_{R}=X_{c}+\frac{W}{2} \\
& Y=Z \alpha+c_{0, v} \frac{Z^{2}}{2}+\not X
\end{aligned}
$$

Equation (1) describes the horizontal profile - the variation of the lateral position $(\mathrm{X})$ of the center of the lane with the distance Z. Equation (4) describes the vertical position for any point on the road. The first two terms compose what we'll call the vertical profile, while the last term is due to the roll angle. All coordinates are given with respect to the car coordinate system, which is placed on the ground in the front center point of the ego vehicle.

\section{LANE DETECTION ALGORITHM}

Lane detection is integrated into a tracking process. The current lane parameters are predicted using the past parameters and the vehicle dynamics, and this prediction provides search regions for the current detection. The detection starts with estimation of the vertical profile, using the stereo-provided 3D information, and afterwards the horizontal profile is detected using a model-matching technique in the image space, and using the knowledge of the already detected vertical profile. The 3D parameters of the horizontal profile are extracted by solving the perspective projection equation. The roll angle is detected last, by checking the difference in height coordinates of the $3 \mathrm{D}$ points neighboring the left and right lane border. The detection results are used to update the lane state through the equations of the Kalman filter.

\section{A. State Prediction}

The lane parameters which have already been presented form the state vector:

$$
\mathbf{X}=\left(W, c_{h, 0}, c_{h, l}, c_{v, 0}, c_{v, l}, X_{c w}, \alpha, \gamma, \psi\right)^{\mathrm{T}}
$$

The uncertainty associated to this vector forms the state covariance matrix $\mathbf{P}$.

The equations presented in [18] are used to obtain the lane state prediction $\mathbf{X}_{\mathbf{p}}$ and its covariance matrix $\mathbf{P}_{\mathbf{p}}$. The addition is the use of a yaw rate sensor (integrated in the ESP system) for measurement of the ego vehicle's curvature, instead of modeling it as noise.

The state prediction is used to obtain the image space prediction. The lane in the image space is represented as a vector $\mathbf{X}_{\mathbf{D}}=\left(x_{l l}, x_{2 b}, \ldots x_{n l}, x_{I n}, x_{2 n} \ldots x_{n r}\right)$, holding the lateral image coordinates ( $x$ coordinates) of the left and right lane borders for fixed $y_{i}$ image coordinates, same as in [3]. This vector has an associated $\mathbf{P}_{\mathbf{D}}$ covariance matrix. The algorithm for generation of $\mathbf{X}_{\mathbf{D}}$ and $\mathbf{P}_{\mathbf{D}}$ from $\mathbf{X}_{\mathbf{P}}$ and $\mathbf{P}_{\mathbf{P}}$ is, however, totally new. This method has been thought as a method for detecting the lane using almost any kind of representation possible.

The first step is to project in the image a series of points $i$ having the coordinates $X i=0, Y i=Y(Z i), Z_{i}=Z_{0}+i D_{Z}$ $Y(Z)$ denotes the equation of the (predicted) vertical profile. The result will be similar to the curve $C$ in the Fig. 1 . The horizontal image lines will be the values of $y_{i}$ for which we'll try to detect the $x$ coordinates. The second step is to project the left and right lane marking points having $Z=Z_{i}$ into the image plane. The $X$ and $Y$ coordinates of these 
points will be found by applying (1), (2), (3) and (4) to the predicted lane parameters $\mathbf{X}_{\mathbf{P}}$. The result of this step are the A points in the Fig. 1. However, the image $x$ coordinate of these points cannot be directly introduced in the $\mathbf{X}_{\mathbf{D}}$ vector. Due to the roll angle and to the fact that the camera may not be horizontally aligned, the A points differ from the points that we seek, which are the B points. The solution is to find the intersection of the $y_{i}$ horizontal lines to the lane border image curves, which are actually composed of line segments. If the value of $D_{Z}$ is accurately tuned to the scenario, this approximation is quite reasonable.

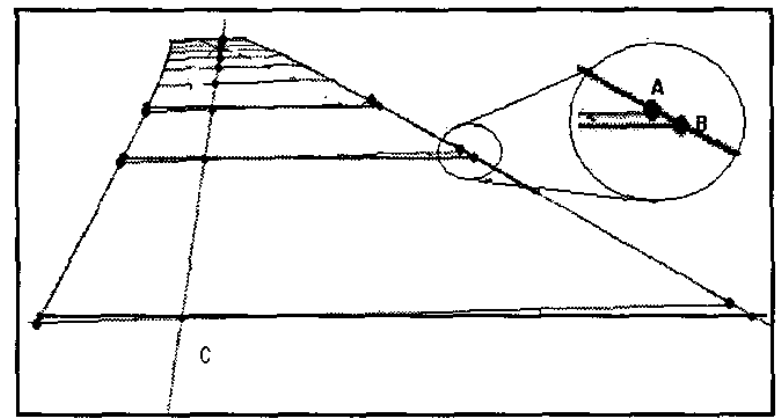

Fig. 1. The lines of constant $Z$ versus the horizontal lines

The values of $\mathbf{P}_{\mathbf{D}}$ are computed using the classical formula of covariance propagation through a linear transformation:

$$
\mathbf{P}_{\mathbf{D}}=\mathbf{J} \cdot \mathbf{P}_{\mathbf{P}} \cdot \mathbf{J}^{\mathbf{T}}
$$

The transformation of $\mathbf{X}_{\mathbf{P}}$ into $\mathbf{X}_{\mathbf{D}}$ is not linear, and cannot even be expressed through a formula. Therefore, the transformation linearization matrix $\mathbf{J}$ (the Jacobian of the transformation) is computed through numerical partial differentiation of each term of $\mathbf{X}_{\mathbf{D}}$ with respect to each term of $\mathbf{X}_{\mathbf{P}}$.

The final result of the prediction is shown in Fig. 2. The vector $\mathbf{X}_{\mathbf{D}}$ provides us the prediction of the lane in the image space, and the diagonal of its covariance matrix $\mathbf{P}_{\mathbf{D}}$ provides the width of the search regions.

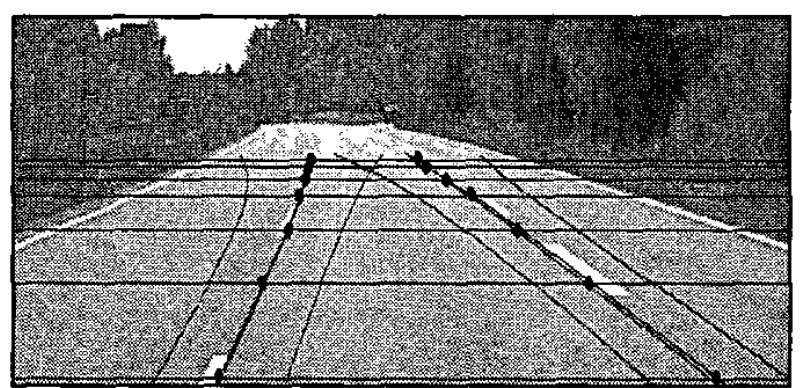

Fig. 2. Results of the lane prediction in the image space

\section{B. Vertical Profile Detection}

The first step of detection is the estimation of the parameters of the vertical profile, which are the pitch angle and the vertical curvature. The side view of the set of $3 \mathrm{D}$ points is taken into consideration, as seen in the Fig. 3. From all the $3 \mathrm{D}$ points only the ones that project inside the predicted search regions are processed.

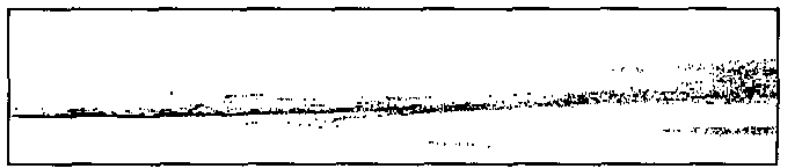

Fig. 3. Side view of the reconstructed 3D points inside the predicted search region

The pitch and vertical curvature are extracted using a method similar to the Hough transform, but instead of having a Hough space of two dimensions, which would complicate the search, we make use of the fact that in the near distance (up to 30 meters) the road can be considered flat. Therefore, an angle histogram is built for each possible pitch angle, using the near $3 \mathrm{D}$ points, and then the histogram is searched from under the road upwards. The first angle having a considerable amount of points aligned to it is taken as the pitch angle. The detection of the curvature follows the same pattern. The pitch angle is considered known, and then a curvature histogram is built, for each possible curvature, but this time only the more distant 3D points are used, because the effect of a curvature is felt only in more distant points.

\section{Horizontal Profile Detection}

The horizontal profile detection is done in the image space, using the search regions generated by the prediction step. The core of the algorithm is a model-matching process similar to the one described in [3], but which works on edges instead of a gradient image. The edge points are filtered - only those edges that correspond to 3D points that comply with the vertical profile are used for lane model matching. The algorithm iteratively matches a straight line inside a search region (a search region is a trapezoid defined by the top and bottom horizontal lines and the width of the bases is given by the diagonal of the covariance matrix $\mathbf{P}_{\mathbf{D}}$ ), and updates the whole $\mathbf{X}_{\mathbf{D}}$ vector through the equations of a Kalman filter. The model is considered fit if enough search regions have been successfully associated. The covariance matrix $\mathbf{P}_{\mathbf{D}}$ contains inside all the constraints of the model (because it was generated through the Jacobian of the projection algorithm), and therefore at the end we'll be sure that what we detect on the image is the perspective projection of the chosen 3D model of the lane.

Now that the lane is detected in the image plane, there is the need of extracting the 3D lane parameters. Again, we must remember that the points that make the $\mathbf{X}_{\mathbf{D}}$ vector do 
not directly correspond to known $Z$ distances. The approach that is taken in order to solve this problem is similar to the approached used for prediction. This time, we project the points of $\left(0, Y\left(Z_{i}\right), Z_{i}\right)$ along with the points $\left(X_{i n f}, Y(Z i)+\gamma\right.$ $\left.X_{i n,}, Z_{i}\right)$, thus generating in the image lines of constant (and known) $Z$. The left and right lane border will consist of line segments, and therefore it is easy to compute their intersection with the lines of constant $Z$. It is the opposite of the prediction (Fig. 1) - now we seek the A points knowing the $B$ points.

It is easy now to extract the $X$ coordinates of the A points. The $Z$ coordinate is known, and the $Y$ coordinate depends on the vertical profile, which is known, and of the roll angle and the $X$ coordinate itself. We also know the image coordinates of the points, and therefore we can solve the perspective projection equation and find $X$. At the end of this step we'll have for each lane border 10 pairs of $(X, Z)$ coordinates. A least-squares technique is used to solve (2) and (3) for the horizontal profile parameters and for the lane width.

\section{Roll Angle Detection}

The roll angle detection becomes straightforward after the image space lane detection. The 3D points that project in the neighborhood of the detected borders are selected. The average height on the left is subtracted from the average height on the right, and this difference is divided by the lane width. The result will be the tangent of the roll angle.

One question raises here: how can we detect the horizontal 3D profile if we haven't detected yet the roll angle? The answer is that the roll angle is one of the parameters of the lane that has a very slow variation in time, and therefore we start initially with a zero roll angle, detect the horizontal profile, and then detect the roll angle, which will be used for horizontal profile detection in the next frame. The values should converge to the correct ones in two or three frames.

\section{E. State Update}

The results of the detection in the current frame are used to update the lane state vector through the Kalman filter, achieving in this way increased stability. In the absence of detection in the current frame the prediction is used as the new state vector, but only up to a point. If the detection lacks for several frames the track is aborted.

\section{RESULTS}

The stereovision-based lane detection system has been tested in a variety of scenarios, mainly on highway roads (Fig. 4) and provincial roads (Fig. 5). The detection has proven fast and accurate. Actually, the algorithm works with almost any kind of lane delimiters (Fig. 6), provided that they obey the clothoid constraints and there are not too many noisy road features (a constraint usually fulfilled by most of the roads). The results are good even in the presence of strong road shadows (Fig.7) or high vertical
(Fig. 6) or horizontal curvatures (Fig.7), or in the presence of obstacles on the current lane (Fig. 8).

The algorithm's performance is decreased in the presence of light saturation, which makes the lane delimiters almost invisible, or in the absence of good camera calibration parameters - a key requirement of any stereovision system.
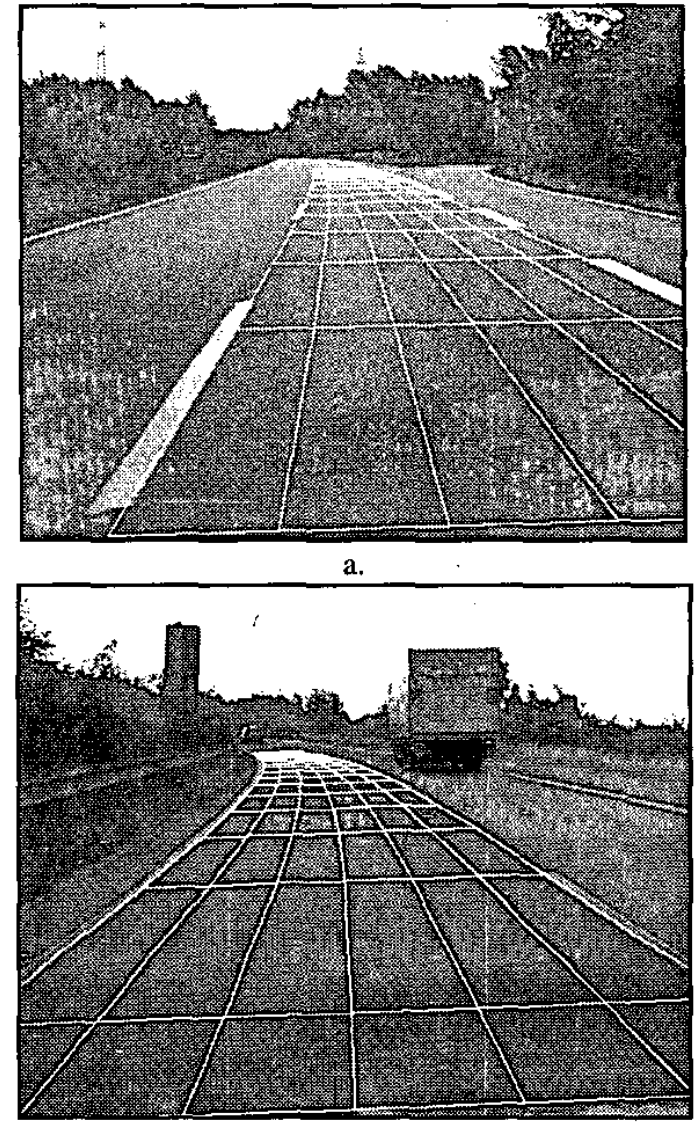

b.

Fig. 4. Results of the lane detection on highways.

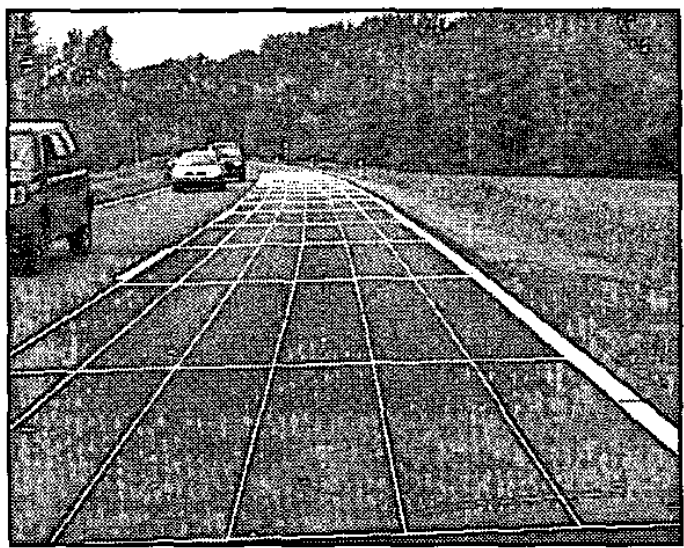

Fig. 5. Results of lane detection on provincial roads. 


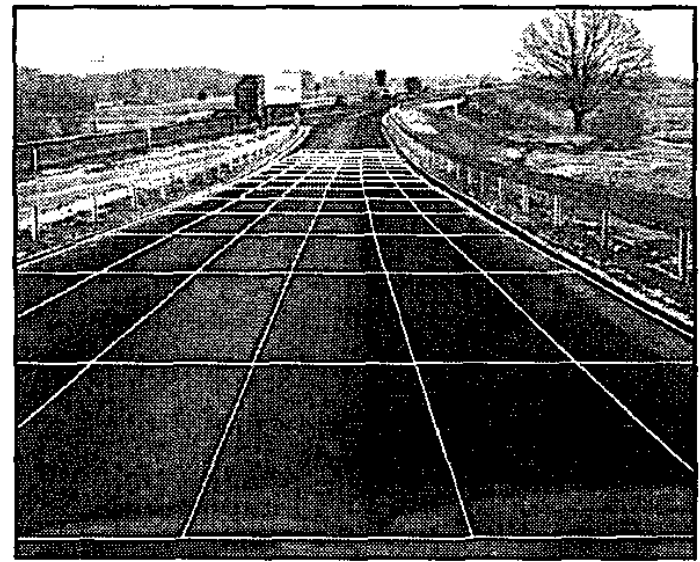

Fig. 6. Results of lane detection on non-flat roads with vertical curvature.

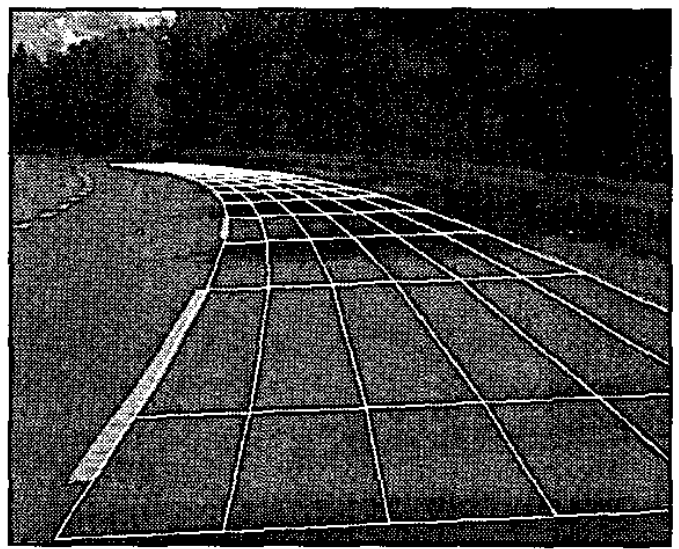

Fig. 7. Results of lane detection on roads with high horizontal curvature and shaded lane markers

\section{CONCLUSIONS}

A technique for lane detection using stereovision has been presented. The method combines several detection and tracking techniques and enhances them for a more accurate and general lane model matching. The lane is represented as a 3D surface, and the assumptions of flat road and zero pitch and roll angles are eliminated due to the availability of the 3D information. This technique can be easily scaled up to another lane model, due to the generality of the model generation and matching and of the 3D parameters reconstruction method.

The results have proven that this is a viable, fast and performant approach. The results can be further enhanced by using supplementary image processing techniques besides edge detection for border feature selection and classification. The use of color information can also add a plus to the robustness and accuracy.

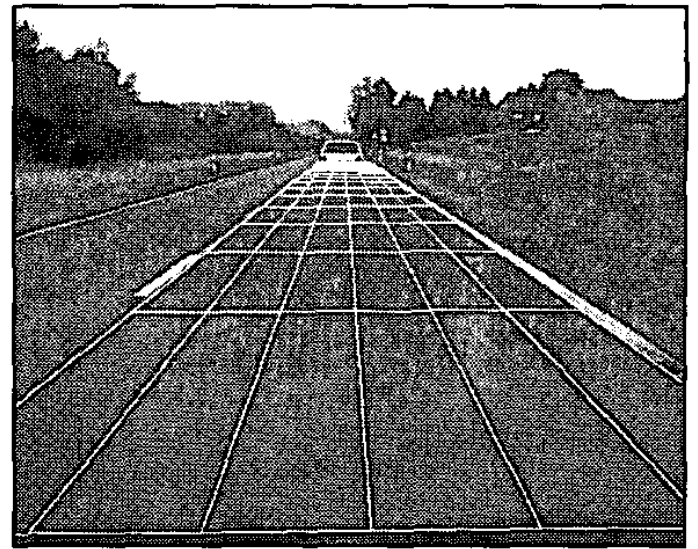

a.

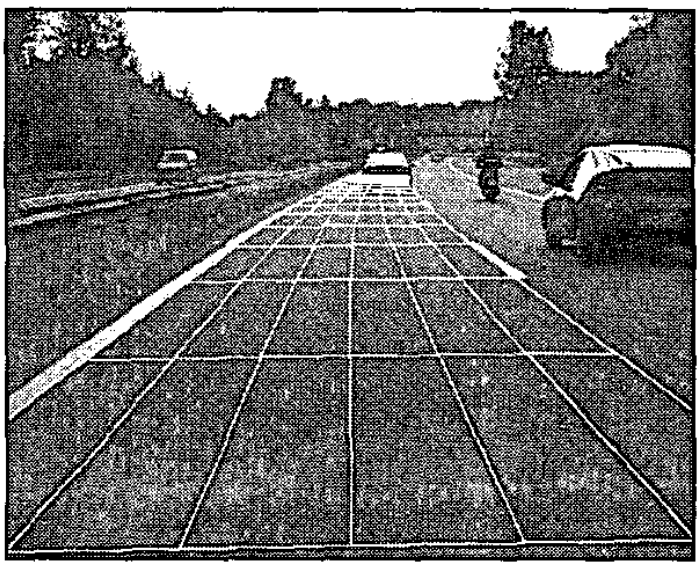

b.

Fig. 8. Results of lane detection the presence of obstacles on the current lane

\section{REFERENCES}

[1] K. Kluge, S. Lankshmanan, "A Deformable-Template Approach to Lane Detection", in Proc. IEEE Intelligent Vehicles' 95, pp. 54-59, Detroit, September 25-26, 1995.

[2] B. Ma, S. Lankshmanan, A. Hero, "Road and Lane Edge Detection with Multisensor Fusion Methods", IEEE International Conf. on lmage Processing, Kobe, Japan, Oct. 1999.

[3] R. Aufrere, R. Chapuis, F. Chausse, "A model-driven approach for real-time road recognition", Machine Vision and Applications, Springer-Verlag, pp. 95-107, 2001

[4] R. Chapuis, F. Marmoiton, R. Aufrere, "Road Detection and Vehicle Tracking by Vision for an On-Board ACC System in the VELAC vehicle", ISIF, 2000

[5] R. Chapuis, R. Aufrere, F. Chausse, "Recovering the 3D shape of a road by vision", Image Processing and its Application" $s$, IEEE, 1999.

[6] F. Chausse, R. Aufrere, R. Chapuis, "Vision based vehicle trajectory supervision", ITSC 2000, Dearborn, Michigan (USA), October 2000, pp. 143-148.

[7] R. Aufrere, R. Chapuis, F. Chausse, "A fast and robust visionbased road following algorithm", IEEE-Intelligent Vehicles Symposium $N 2000$, Dearborn, Michigan (USA), October 2000, pp. 192-197.

[8] B. Yu, A. K. Jain, "Lane Boundary Detection Using A Multiresolution Hough Transform", International Conference 
on Image Processing (ICIP '97), 3-Volume Set-Volume 2 October 26 - 29, 1997 Washington, DC, p. 748.

[9] Jung Kang, J. Won Choi and In So Kweon, "Finding and Tracking Road Lanes using Line-Snakes" in Proceedings of Conference on Intelligent Vehicles, pp. 189-194, 1996, Japan.

[10] Y. Wang et al., "Lane detection using spline model", Pattem Recognition Letters vol. 21 (2000), no. 8, pp. 677-689.

[11] A. Broggi, "Robust Real-Time Lane and Road Detection in Critical Shadow Conditions", In Proceedings IEEE International Symposium on Computer Vision, 1995, pp.353358

[12] M. Bertozzi, A. Broggi, A. Fascioli, and A. Tibaldi, "An Evolutionary Approach to Lane Markings Detection in Road Environments", In Atti del 6 Convegno dell'Associazione Italiana per l'Intelligenza Artificiale, pages 627-636, Siena, Italy, September 2002.

[13] G.Y. Jiang; T.Y. Choi, S.K. Hong; J. W. Bae, B. S. Song, "Lane and obstacle detection based on fast inverse perspective mapping algorithm", IEEE International Conference on Systems, Man, and Cybernetics, Vol. 4, 2000.

[14] S. T. Park et al., "Real-Time Lane Recognition by Simulated annealing algorithm", 1999.

[15] A. Kreucher, S. Lanksmanan, "LANA: A Lane Extraction Algorithm that Uses Frequency Domain Features", IEEE Transactions on Robotics and Automation, Volume: 15 Issue: 2, Apr 1999, pp. 339-345.

[16] J.P. Gonzalez, U. Ozguner, "Lane Detection Using HistogramBased Segmentation and Decision Trees" in Proc. JEEE-ITS 2000 , pp. 346-351

[17] A. Takahashi, Y. Ninomiya, "Model-Based Lane Recognition", Proceedings of the IEEE Intelligent Vehicles Symposium, 1996, 201-206.

[18] J. Goldbeck, B. Huertgen, "Lane Detection and Tracking by Video Sensors", In Proc. IEEE International Conference on Intelligent Transportation Systems, October 5.8, 1999, Tokyo Japan, pp. 74-79. 